\title{
Notch-Mediated Polarity Decisions in Mechanosensory Hair Cells
}

\author{
A. Jacobo ${ }^{1, *,}$, A. Dasgupta ${ }^{1, \dagger}$, A. Erzberger ${ }^{1}, K^{\prime}$. Siletti ${ }^{1,2}$, and A. J. Hudspeth ${ }^{1}$ \\ ${ }^{1}$ Howard Hughes Medical Institute and Laboratory of Sensory Neuroscience, The Rockefeller Uni- \\ versity, New York, NY 10065,USA. \\ 2 Present address: Department of Medical Biochemistry and Biophysics, Karolinska Institutet, Stockholm, \\ Sweden \\ * To whom correspondence should be directed at <ajacobo@rockefeller.edu $>$. \\ $\dagger$ These authors contributed equally to this work.
}

November 27, 2018

The development of mechanosensory epithelia, such as those of the auditory and vestibular systems, results in the precise orientation of mechanosensory hair cells and consequently directional sensitivity. After division of a precursor cell in the zebrafish's lateral line, the daughter hair cells differentiate with opposite mechanical sensitivity. Through a combination of theoretical and experimental approaches, we show that Notch1a-mediated lateral inhibition produces a bistable switch that reliably gives rise to cell pairs of opposite polarity. Using our mathematical model of the process, we predict the outcome of several genetic and chemical alterations to the system, which we then confirm experimentally. We show that Notch1a downregulates the expression of Emx2, a transcription factor known to be involved in polarity specification, and acts in parallel with the planar-cell-polarity system to determine the orientation of hair bundles. By analyzing the effect of simultaneous genetic perturbations to Notch1a and Emx2 we infer that the generegulatory network determining cell polarity includes undiscovered polarity effectors.

\section{Introduction}

Notch-Delta signaling regulates diverse processes in development, regeneration, and disease. In many contexts signaling through this pathway creates cellular patterns through suppressive cell-cell interactions, in which the concentrations of signals in interacting cells diverge over time, leading to distinct cellular fates $(1-3)$. We have discovered a novel role for Notch-mediated lateral inhibition in determining the polarity of sensory hair cells during development and regeneration.

By transducing mechanical stimuli into electrical signals, hair cells underlie our senses of hearing and balance (4). The polarity of a hair cell is morphologically determined by its hair bundle, which protrudes from the cell's apical surface and confers directional sensitivity to mechanical stimuli (5). Although signaling by the core planar-cell-polarity (PCP) system aligns hair bundles with the polarity axis of a sensory $\operatorname{organ}(6,7)$, some hair cells reverse their polarity with respect to the PCP axis, rendering them optimally responsive to mechanical stimuli from the opposite direction $(8,9)$. The transcription factor Emx2 is specifically expressed in hair cells with reversed polarity and participates in the specification of that fate (10), but the mechanism that establishes the Emx2 identity of a hair cell and effects polarity reversal remains unknown.

Using the lateral line of the zebrafish as a model system, we have studied how the correct number of oppositely polarized hair cells is established during the formation of mechanosensory epithelia. The lateral line consists of a series of sensory organs 
bioRxiv preprint doi: https://doi.org/10.1101/480798; this version posted November 28,2018 . The copyright holder for this preprint (which was not certified by peer review) is the author/funder, who has granted bioRxiv a license to display the preprint in perpetuity. It is made available under aCC-BY-ND 4.0 International license.

Notch-Mediated Polarity Decisions in Mechanosensory Hair Cells
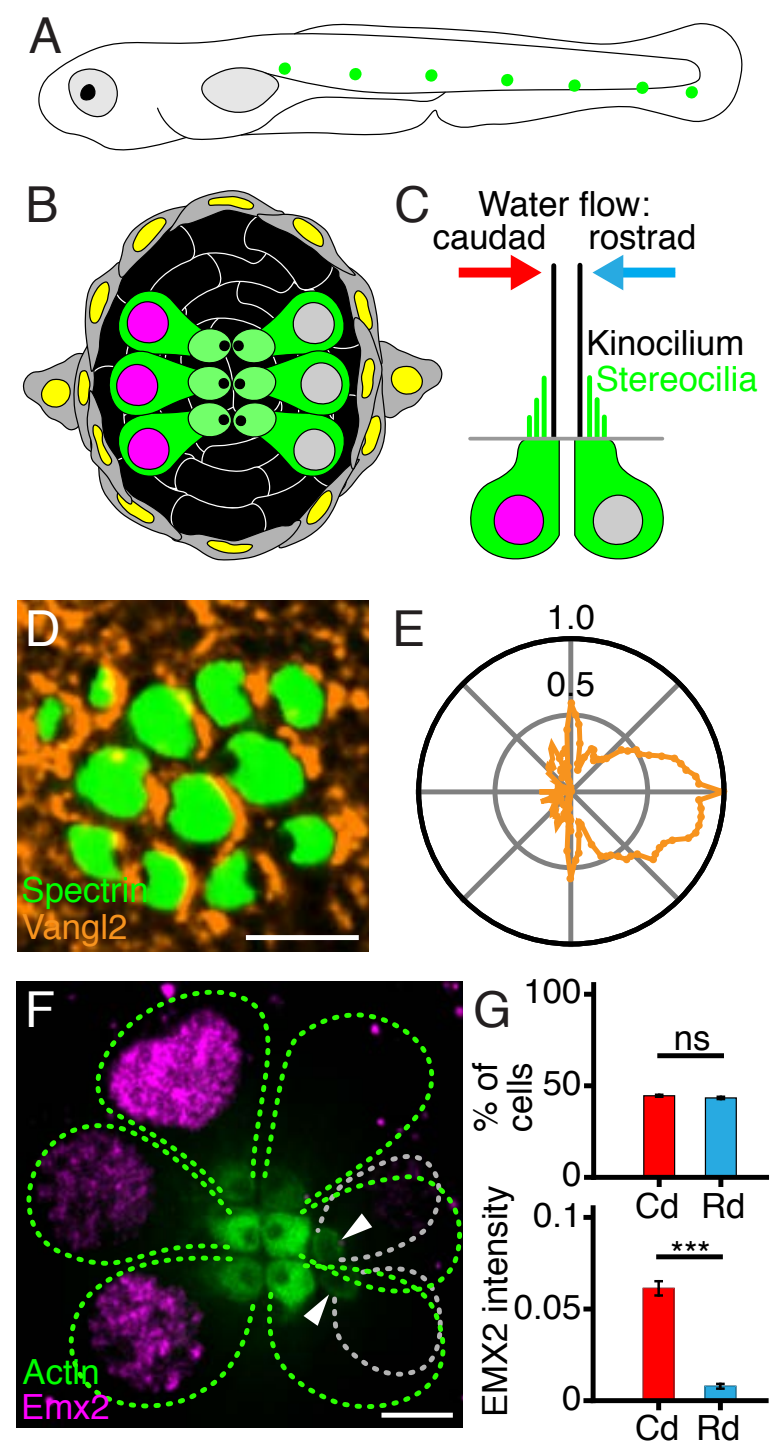

called neuromasts that sense water movements (Figure 1A) (9). Hair cells in the neuromast develop in pairs from a progenitor cell, with the two daughter cells bearing oppositely polarized hair bundles (9). One cell is sensitive to caudad water motion-flow toward the tail-whereas the sibling cell responds to rostrad movement-flow toward the head (Figure $1 B, C)$. The expression of the core PCP protein Vangl2 is indistinguishable in the two cells (Figure $1 \mathrm{D}, \mathrm{E})$, but only caudad-polarized cells express Emx2 (Figure 1F,G) (10). Here we demonstrate that lateral inhibition mediated by Notch-Delta signaling dictates the binary fate decision involving Em×2 that underlies polarity patterning.
Figure 1: Organization of neuromasts in the zebrafish's lateral line. (A) A schematic drawing of a 4 dpf larva depicts neuromasts (green) of the posterior lateral line. Additional neuromasts (not shown) adorn the anterior portion of the larva. (B) A diagramatic surface view of a single neuromast shows hair cells (green) separated by supporting cells (black) and surrounded by mantle cells (gray). (C) A schematic section depicts the sensitivity of oppositely oriented hair cells to water flow. (D) In a micrograph of the upper surface of a neuromast, spectrin (green) marks the cuticular plates of hair cells. Immunolabeling reveals that the core PCP protein Vang/2 (orange) occurs consistently at the posterior cellular boundaries. (E) A polar plot of average Vangl2 labeling from 502 hair cells quantitates the effect in panel $\boldsymbol{D}$. This and each of the subsequent polar plots represents the average intensity of labeling, normalized to the maximum value, as a function of angular position with respect to the cells' centers (Supplementary Material). (F) In a developing neuromast, Em×2 (magenta) is expressed in the nuclei of three mature hair cells (green dashed lines on the left). In the distribution of actin-GFP (green) at the cellular apices, the dark spots locate the kinocilia and indicate that those cells are sensitive to caudad stimulation. Three other mature hair cells (green dashed lines on the right) have an opposite orientation. The apices of two immature hair cells (gray dashed lines) are indicated by arrowheads. (G) The polarities of 81 hair cells from 18 neuromasts (top) are equally divided between caudad (Cd) and rostrad (Rd). The former cells express significantly higher levels of Emx2 (bottom). Scale bars, $3 \mu \mathrm{m}$. Means $\pm S D s ;{ }^{* * *}=\mathrm{p}<0.001$; $n s=$ not significant.

\section{Results}

\section{Notch-mediated lateral inhibition and po- larity reversal}

Pharmacological or genetic interference with Notch signaling yields neuromasts with a polarization bias of their hair cells $(11,12)$. This observation raises the possibility of an interaction between the Notch pathway and $E m \times 2$ in the establishment of polarity reversals. To explore this hypothesis, we developed a mathematical model of Notch signaling between a pair of nascent hair cells based on the framework in Boareto et al. (Figure 2A; Supplementary Information) (13). We simulated the concentration dynamics of ligands and receptors on the cellular surfaces as well as the concentration of Notch intracellular domain (NICD), which is released by proteolytic cleavage after the interaction of Notch with the lig- 
and Delta and acts as a transcriptional co-activator. We used realistic kinetic rates for binding, unbinding, receptor cleavage, and nuclear translocation (Supplementary Table S2; Supplementary Material) $(13,14)$.

Trans-activation of the Notch receptor inhibits the expression of its ligand Delta, creating a negative feedback loop (Figure 2A). Although our simulations started with similar concentrations of NICD in each of the two cells, feedback therefore amplified the initial stochastic differences of NICD expression in the nascent hair-cell pair and broke the initial symmetry (Figure 2A,B). One of the cells evolved to a low-Notch and high-Delta phenotype called the sender state (14). That cell suppressed the expression of Delta in its sister, which then achieved a high-Notch phenotype termed the receiver state (Figure 2B). Given that the expression of Emx2 also shows distinct levels in sister cells (Figure $1 F, G$ ), we propose that Notch signaling regulates the expression of Emx2, giving rise to a pair of hair cells of which one expresses a high and the other a low concentration of this protein. This arrangement reliably creates one cell with caudad orientation and another with rostrad orientation (Supplementary Material).

To test our model, we investigated the expression of the Notch ligands Delta C and Delta D in regenerating neuromasts of zebrafish larvae at four days post-fertilization ( $4 \mathrm{dpf}$ ). Nascent hair cells that had recently originated from a progenitor cell expressed higher levels of Delta D than mature hair cells with fully formed hair bundles. These nascent cells neither possessed an apical surface nor expressed Em×2. In slightly older but still immature pairs of hair cells, defined as those with underdeveloped apical surfaces, Delta $\mathrm{D}$ occurred at the contact area between the cells and was sometimes asymmetrically enriched in the cell that expressed Emx2 (Figure 2C; Supplementary Figure 1A-C; Supplementary Movies 1-3). Furthermore, we quantified the expression of Delta $D$ and $E m \times 2$ in nascent, immature, and mature haircell pairs and found that Delta $\mathrm{D}$ expression preceded that of Emx2 (Figure 2D). This finding suggests that Notch signaling anticipates the expression of Emx2 during polarity specification. During the maturation of a hair-cell pair the sender cell, with the higher Delta concentration and significant $E m \times 2$, has a low Notch concentration. The results suggest that Notch downregulates Emx2 expression and support the hypothesis that Notch-mediated lateral inhibition breaks the symmetry between the two nascent hair cells and defines their Emx2 status. Moreover, the observations imply that the sender cell is destined to develop caudad polarization and the receiver cell to assume rostrad polarization.

\section{Perturbations of Notch gene expression}

If Emx2 expression is regulated through Notch signaling, our model provides two predictions for experimental testing. First, in the neuromasts of a Notchknockout animal, all hair cells should be Em×2positive and display caudad sensitivity. And second, constitutive expression of NICD in hair cells should yield two high-Notch, low-Emx2 cells with a rostrad polarity.

To experimentally test the first prediction of our model, we analyzed neuromasts from larvae deficient in functional Notch proteins. In homozygous Notch1ab420-/- mutants (15) we found that all hair cells were Emx2-positive and displayed a caudad polarity (Figure 2E,F; Supplementary Figure 2A). By contrast, homozygous Notch2el515-/- mutants (16) showed no polarity bias: either Notch2 is not involved in polarity regulation or Notch1a compensates for its absence (Supplementary Figure 2B). We additionally examined neuromasts from $\operatorname{delta} A^{-/-}$, delta $C^{-/-}$, and delta $D^{-/-}$mutants $(17,18)$ and observed no bias in hair-cell polarity (Supplementary Figure 2B). Although the asymmetrical localization of Delta $D$ in immature hair-cell pairs suggests that the protein participates in lateral inhibition, the lack of polarity bias in Delta D mutants points to compensation through functional redundancy among the three ligands.

To test our second prediction we developed a transgenic zebrafish line that constitutively expresses myctagged NICD (NICD-CE) specifically in hair cells (Supplementary Table S1; Supplementary Material). As reported previously (12), hair cells of these transgenic larvae display a strong rostrad bias compared to those in control animals (Figures $1 G$ and $2 G$ ). To investigate the mechanism behind this bias, we used specific antibodies to analyze the $\mathrm{Em} \times 2$ level in NICD-CE hair cells. Because expression was sometimes mosaic, we restricted our analysis to hair cells expressing NICD-CE as indicated by antimyc immunolabeling (Figure 2G). Quantification of nuclear expression of $E m \times 2$ in both control and NICD-CE hair cells revealed that NICD decreased the expression of Emx2, resulting in rostrad polar- 

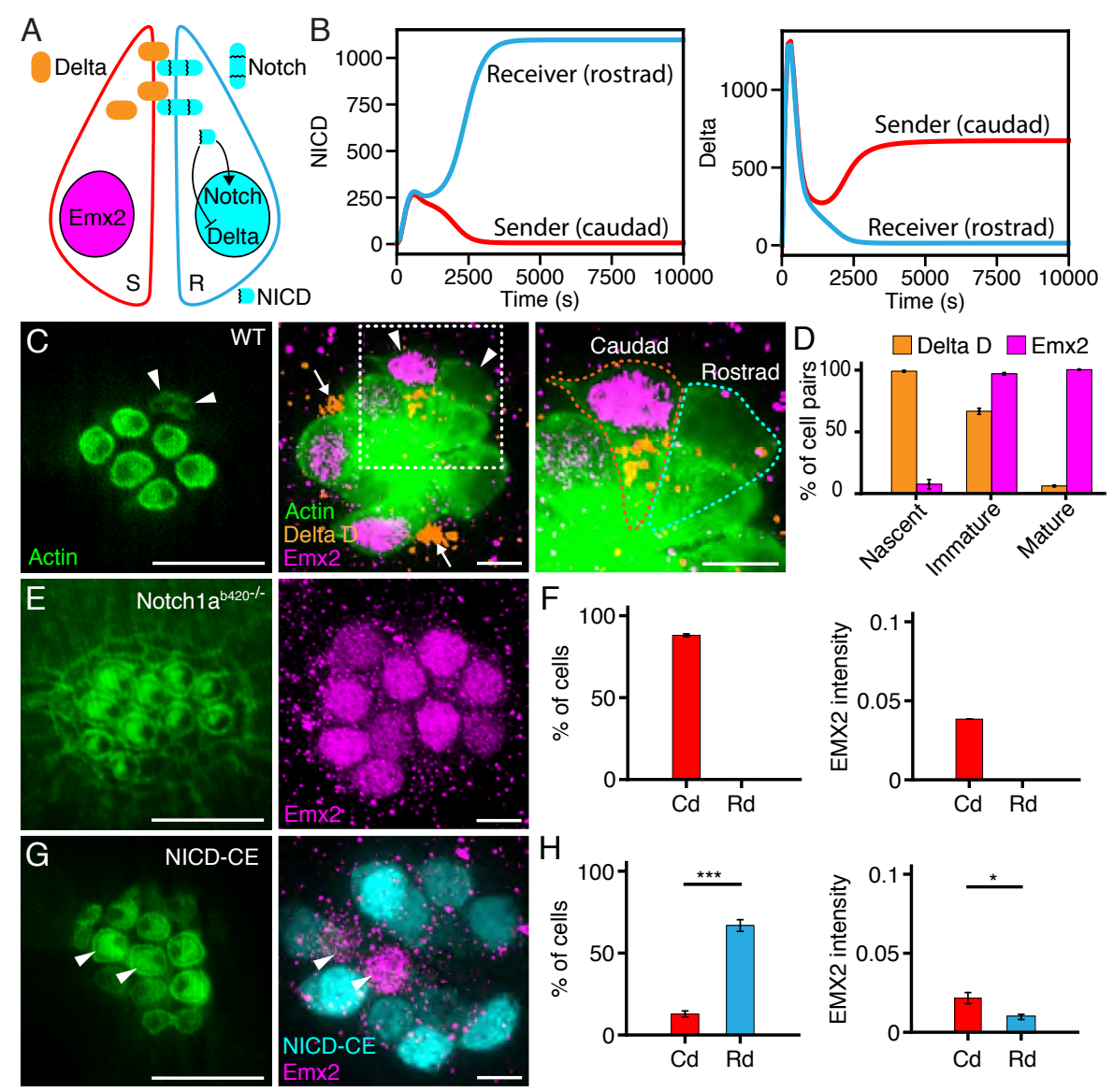

Figure 2: Notch-mediated lateral inhibition and hair-cell polarity. (A) A schematic representation of our Notch signaling model shows a pair of nascent hair cells where the Delta ligand (orange) expressed on one cell causes cleavage of Notch receptor (blue) on the other. Upon entering the nucleus, NICD upregulates Notch expression, downregulates Delta expression, and conduces to a receiver phenotype $(R)$. The sister cell evolves in the opposite direction to a sender fate $(S)$. (B) Representative simulations portray the concentrations of NICD (left) and Delta (right) in the receiver cell-which becomes rostrad-polarized-and the sender cell-which becomes caudad-polarized. (C) An apical view of a 4 dpf wild-type neuromast (left) reveals three mature pairs of hair cells and one immature pair (arrowheads). A view at the nuclear level (center) shows immunolabeling of the same neuromast for Emx2 (magenta) and Delta $D$ (orange). An enlargement of the boxed area (right) includes outlines of the two immature hair cells. Hair-cell progenitors also express Delta D (arrows). (D) Delta D is expressed in at least one hair cell of each pair at earlier stages of development than Emx2 (51 nascent pairs, 101 immature pairs, 259 mature pairs). (E) In a Notch1ab420-/- mutant, hair cells show a caudad polarization (left) and uniformly express Emx2 (right). (F) These effects are consistent in 536 hair cells from 57 neuromasts. (G) In an NICD-CE transgenic animal, most hair cells are sensitive to rostrad stimulation (left). Occasional hair cells (arrowheads) fail to express NICD-CE; they instead contain Emx2 (magenta, right) and adopt a caudad sensitivity. (H) The effect is quantified for 68 cells from 21 neuromasts. Scale bars, $5 \mu \mathrm{m}$. Means $\pm S D s ; * * *=p<0.001 ; *=p<0.02$.

ity (Figure 2H). We corroborated this result with another transgenic line that induced endogenous production of NICD through cytokine signaling (Supplementary Figure 3 A,B; Table S1; Supplementary Material). Taken together, measurements of polarity distribution and Emx2 expression levels in mutant and transgenic lines show that Notch regulates polarity reversal.

\section{Notch-Delta signaling and global PCP sig- nals}

Because Notch signaling determines Em×2 expression in developing pairs of hair cells, we wondered how the Notch pathway interacts with cell-intrinsic polarity factors and with global PCP signals to determine the orientation of hair bundles. In trilobite mutants that lack functional Vangl2 protein, hair bundles are randomly oriented (7). Because Emx2 is nevertheless expressed in half of the Vang/2-/- 
mutant hair cells (Supplementary Figure 4) (10), though, a Notch-based lateral-inhibition circuit remains intact in Vang/2-/- mutants and might act in parallel to the PCP system.

The shape and orientation of the hair bundle is determined by the localization of cell-intrinsic polarity factors including the heterotrimeric G-protein Gnai $(19,20)$. Reading cues from the global PCP pathway, Gnai and the associated proteins form a crescent-shaped domain at the apical surface of a developing mammalian hair cell. These factors are implicated in relocalizing the kinocilium from its initial position at the center of the apical surface to the edge where the Gnai crescent lies $(19,20)$. This process creates a cell-intrinsic polarity axis that defines the orientation of the hair bundle. Whereas at least three isoforms of Gnai occur in the zebrafish genome, RNA-seq data indicate that Gnai1 is highly enriched in lateral-line hair cell (21). Regardless of the cellular polarity, Gnail adorned the apical surface of each wild-type hair cell as a crescent adjacent to the kinocilium (Figure 3A). Even in immature hair cells with an underdeveloped apical surface, Gnai was asymmetrically distributed, suggesting that Gnai plays a role similar to that of its mammalian homolog.

We quantified the average intensity of Gnai1 expression across the apical surfaces of hair cells and observed a mirror-symmetric distribution (Figure 3B). This opposing pattern of localization was lost in Notch1a $a^{b 420-/-}$ mutants (Figure 3C,D) and NICD-CE larvae (Figure 3E,F), in which the distribution of Gnail nonetheless coincided with the orientation of the hair bundles. The mirror symmetry of the Gnai1 distribution was also absent from $E m \times 2^{-/-}$ mutants and larvae constitutively expressing $E m \times 2$ (Em×2-CE) (Supplementary Figure 4 A,B; Table S1) (10). Notch and Emx2 therefore act upstream of the cell-intrinsic determinants of hair-cell polarity and guide their localization during development.

To further investigate whether core PCP components were affected in Notch-deficient and NICD-CE larvae, we analyzed the localization of Vangl2 protein. In wild-type larvae, Vangl2 was enriched at the posterior edge of a hair cell's apical surface irrespective of the bundle's orientation (Figure 1D,E). This expression pattern was maintained in the neuromasts of both Notch1ab420-/- mutants (Figure 3G,H) and NICD-CE larvae (Figure 3I,J). A similar pattern also occurred in $E m \times 2^{-/-}$mutants and $E m \times 2-C E$ transgenic larvae (Supplementary Figure 4C,D). Because the distribution of Vangl2 was unaffected by a deficit or excess of Notch function, Notch signaling operates in parallel to the core PCP pathway.

\section{Interaction of Notch and Emx2 with down- stream targets}

Emx2 is necessary and sufficient to determine the polarity of hair cells $(10)$. Because our observations suggested that Em $\times 2$ functions downstream of Notch, expression of both NICD and Em×2 would be expected to yield hair cells with caudad sensitivity similar to those in Em×2-expressing larvae. We therefore developed a line of transgenic zebrafish that constitutively expressed both Em×2 and NICD within hair cells (Figure 4A; Supplementary Figure 5; Supplementary Movie 6; Supplementary Table S1). Surprisingly, hair cells displayed a rostrad polarity bias similar to that characteristic of NICD-CE alone (Figure 4B). This result implies that Emx2 and Notch interact in a more complex manner than the simple downregulation of $E m \times 2$ by Notch. We found that in $E m \times 2-C E$ hair cells the endogenous $E m \times 2$ retained a pattern of high expression in one sister cell and low expression in the other (Supplementary Figure 6), suggesting that there is no feedback regulation of the Notch pathway through Em×2.

To explain these results, we extended our mathematical model to include an additional hypothesis: NICD and Emx2 compete for the regulation of genes that encode one or more polarity effectors. We propose that $E m \times 2$ activates a polarity effector and NICD inhibits it (Figure 4C; Supplementary Information). By symmetry, the model would also operate if these roles were reversed. The concentration of the polarity effector establishes cellular polarity: high concentrations yield caudad-polarized cells whereas low concentrations produce cells of rostrad sensitivity. Under these assumptions our model reproduced the results of all the experimental perturbations that we had performed, including the constitutive expression of both NICD and Emx2 (Figure 4D-I). By including noise in the model we were also able to quantitatively reproduce the polarity biases observed experimentally (Figure 2E-H; Figure 4A,B; Supplementary Figure 8A,B).

In wild-type neuromasts the lateral-inhibition mechanism splits sister cells into two states, low-

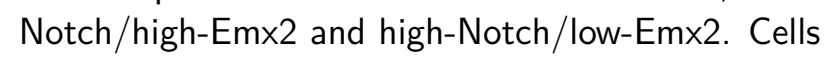


bioRxiv preprint doi: https://doi.org/10.1101/480798; this version posted November 28, 2018. The copyright holder for this preprint (which was not certified by peer review) is the author/funder, who has granted bioRxiv a license to display the preprint in perpetuity. It is made available under aCC-BY-ND 4.0 International license.

Notch-Mediated Polarity Decisions in Mechanosensory Hair Cells
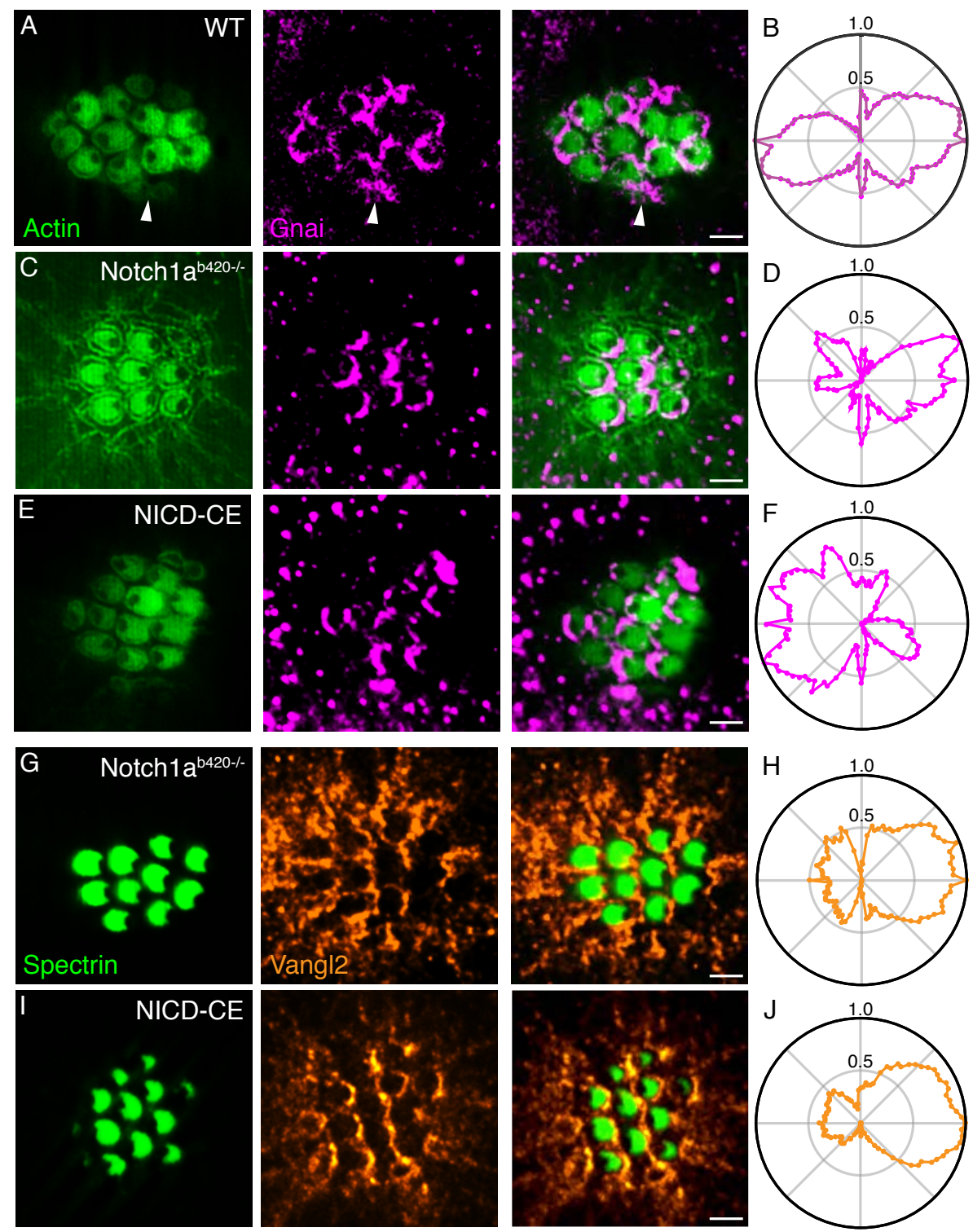

Figure 3: Parallel action of Notch and PCP. (A) In an apical view of a wild-type neuromast, hair-cell polarity is delineated by actin-GFP (green, left) and Gnai is immunolabeled (magenta, center). As shown in the merged view (right), Gnai already displays a polarized location in immature hair cells (arrowheads). (B) In the average radial intensity profile of Gnai from 239 hair cells of both polarities, caudad and rostrad cells create symmetric lobes on the two halves of the apical surface. (C) Notch1 $\mathrm{a}^{\mathrm{b} 420-/-}$ hair cells display a consistently caudad orientation and crescents of Gnai at their posterior boundaries. (D) A polar plot from 81 hair cells confirms the asymmetrical distribution of Gnai. (E) NICD-CE hair cells are consistently sensitive to rostrad stimulation and bear Gnai at their anterior edges. (F) A polar plot from 35 hair cells confirms the asymmetry of Gnai distribution. (G) Immunolabeling for spectrin (green, left) shows that Notch1a ${ }^{\text {b420-/- }}$ hair cells have caudad sensitivity; labeling for Vang/2 (orange, center) displays its distribution at the posterior edges of the cells. $(\boldsymbol{H})$ A polar plot from 155 hair cells confirms the asymmetrical distribution of Vang/2. (I) Although NICD-CE hair cells are consistently sensitive to rostrad stimulation, they also bear Vangl2 at their posterior edges. (J) A polar plot from 235 hair cells confirms the asymmetry. Scale bars, $2 \mu \mathrm{m}$.

with low Notch and high Emx2 concentrations drive the expression of the polarity effector above a certain threshold and become caudad-polarized, whereas in cells with high Notch and low Emx2 concentrations the expression of the polarity factor is repressed and they become rostrad-polarized (Figures $1 \mathrm{G}$ and 4D; Supplementary Figure 8).
The first indication that Notch plays a role in regulating polarity reversals was the observation that treatment with $\mathrm{N}$-[N-(3,5-difluorophenacetyl-L-alanyl)]S-phenylglycine $t$-butyl ester (DAPT), a $\gamma$-secretase inhibitor that prevents the liberation of NICD, produced neuromasts with a rostrad bias (Supplementary Figure 9) $(11,22)$. Because DAPT blocks 
Notch activity, treated neuromasts might be expected to show a caudad bias similar to that of Notch1ab420-/- mutants. The seemingly paradoxical result can be explained in light of our model by assuming that DAPT inhibits Notch cleavage incompletely. In this case the bistable circuit that generates high- and low-NICD states fails for many nascent hair-cell pairs. Both cells adopt an intermediate level of NICD that nonetheless suffices to repress $E m \times 2$ and then to drive the expression of the polarity effector to sub-threshold levels (Figure 4E; Supplementary Figure 9; Supplementary Information). If the activity of Notch were inhibited more completely, our model predicts that cells would show a caudad bias. Although the regime of low NICD expression was inaccessible through the use of higher concentrations of the inhibitor, which proved lethal to larvae, it could be studied in Notch1ab420-/- mutants. Here all cells evidently adopted a high-Emx2 state, which drove the expression of the polarity effector above threshold and rendered them caudadpolarized (Figure 4F). Conversely, when NICD was expressed constitutively, all cells adopted a high$\mathrm{NICD} /$ low-Emx2 state, inhibiting the polarity effector and becoming rostrad-polarized (Figure 4G; Supplementary Figure 10).

When Emx2 was expressed constitutively, the bistable circuit producing high- and low-NICD cells remained intact, and therefore cells assumed a low-

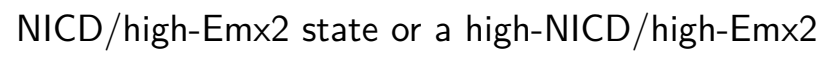
state (Figure $4 \mathrm{H}$ ). In this case the constitutive expression of Emx2 was able to drive the expression of the polarity effector even in high-NICD cells, rendering them caudad-polarized. If NICD and Em×2 were expressed simultaneously then all cells adopted a high-NICD/high-Emx2 state in which repression of the polarity effector by NICD overcame the activation by $\mathrm{Em \times 2}$ and produced a rostrad bias (Figure 4I). Although either treatment with DAPT or simultaneous constitutive expression of NICD and Em $\times 2$ produces a similar rostrad bias, our model predicts different mechanisms for these effects (Figure 4B; Supplementary Figure 9B). In the case of DAPT treatment we expect that most hair-cell pairs have opposite polarities, with a minority of pairs of rostrad polarity (Supplementary Information). For simultaneous constitutive expression of NICD and Emx2, however, most pairs demonstrate the same polarity, with a bias towards rostrad polarization (Supplementary information). By examining the polarity of hair cells known to be sisters, we confirmed this prediction (Supplementary Figure 11).

Our model thus explained the polarity biases observed in different experimental perturbations in terms of the cells' joint Emx2 and NICD concentrations and the effect of these proteins on a downsteam effector of polarity reversal.

\section{Discussion}

The coordination of polarity reversals through lateral inhibition produces pairs of oppositely polarized hair cells with virtually no errors. Our results show that robustness is ensured by the gene-regulatory network of the Notch signaling pathway, in which the downregulation of Delta ligands by NICD creates a negative feedback loop between each pair of nascent hair cells and leads to the emergence of a bistable switch (Supplementary Information) $(13,14)$. This switch reliably breaks the symmetry between the two nascent hair cells and drives them to distinct states of Notch expression. When NICD is constitutively expressed both cells downregulate Emx2 and develop rostrad-polarized hair bundles. In Notch1a $a^{b 420-/-}$ mutants, Em×2 is upregulated in hair-cell pairs and renders them caudad-polarized. In conjunction with mathematical modelling, experiments in which NICD and Em×2 are expressed simultaneously suggest that Notch and Emx2 compete for the regulation of a polarity effector that institutes reversal of the hair-bundle orientation. Studying this effector will be the aim of future research.

To the best of our knowledge, polarity determination in the neuromast represents the first phenomenon in which the PCP and Notch pathways act in parallel to determine the orientation of a subcellular structure. The PCP pathway specifies an axis for the polarization of hair bundles; the Notch pathway then coordinates the reversal of half the bundles with respect to that axis. This process creates neuromasts in which all the cells are aligned with the anteroposterior axis of the fish, but half point in the opposite direction. This arrangement confers sensitivity to both rostrad and caudad water flows. By elucidating the interplay between cell polarity and Notch signaling, our findings explain how a complex polarity pattern is formed robustly during development and regeneration. 

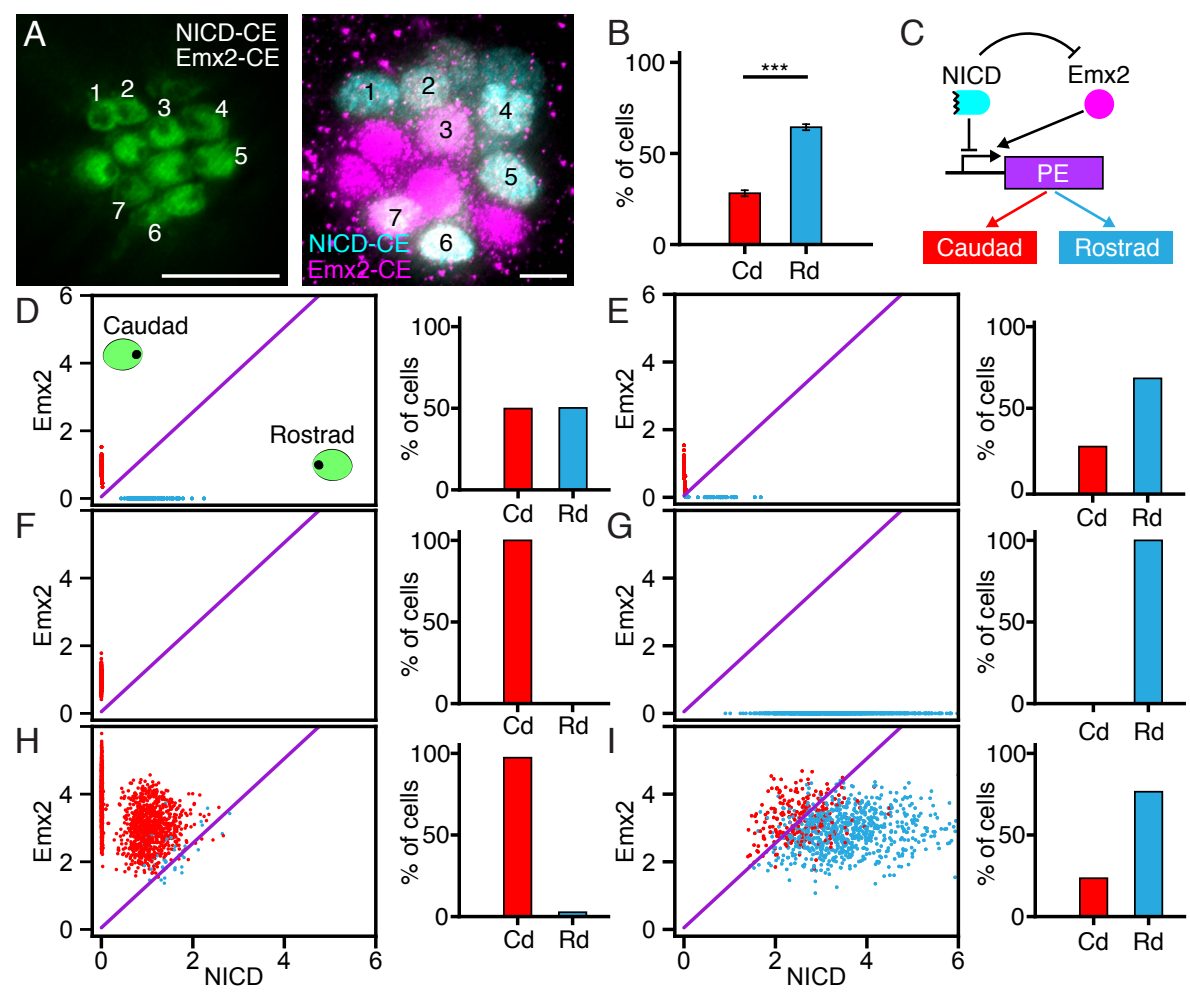

Figure 4: Figure 4. Modeling of the regulatory network underlying hair-cell polarity. (A) In an apical view, numerals mark the hair cells in a larva constitutively expressing both NICD (cyan) and Emx2 (magenta). (B) In larvae expressing both proteins, 334 hair cells from 50 neuromasts display a strong bias toward rostrad polarity. (C) In the proposed regulatory network, Notch and Emx2 control hair-cell polarity through a downstream polarity effector $(P E)$, which might be a single protein or a more complex system. (D-I) The panels display the steady state expression of Emx2 and NICD for multiple simulations of hair-cell pairs of (D) wild-type, (E) DAPT-treated, (F) Notch1a ${ }^{/ /}$,

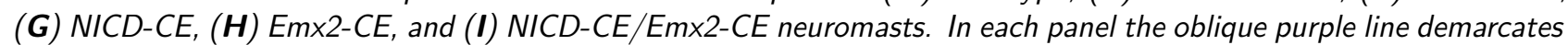
the threshold for switching of the polarity effector. Scale bars, $5 \mu \mathrm{m}$. Means $\pm S D$ s; $* * *=p<0.001$.

\section{Acknowledgments}

We thank Anna Kaczynska for expert fish husbandry and the members of our research group for comments on the manuscript. Lilianna Solnica-Krezel (Washington Univ, St. Louis) kindly provided rabbit anti-Vangl2 antiserum; Ivana Mirković and Sergiy Pylawka developed the $\mathrm{Tg}$ (myo6b:GAL4FF) line; and Sharon Amacher (Ohio State University), David Trevor (University of California, San Diego), and Kenneth Wallace (Clarkson University) provided various strains of zebrafish. A.J. was supported by an F.M. Kirby Postdoctoral Fellowship from Rockefeller University, A.E. by a Feodor Lynen Fellowship from the Alexander von Humboldt Foundation, and K.S. by Ruth L. Kirschstein National Research Service Award DC014212 from the National Institute on Deafness and Other Communication Disorders. A.D is a Postdoctoral Associate and A.J.H. an Investigator of Howard Hughes Medical Institute.

\section{Conflict of interest}

The authors declare no conflicts of interest.

\section{Authors' contributions}

A.J. initiated the project, conducted experiments, developed the theoretical model, quantified the results, and wrote the paper; A.D. conducted experiments, quantified the results, and wrote the paper; A.E. conducted image analysis, quantified the results, and wrote the paper; K.S. initiated the project and conducted experiments; and A.J.H. wrote the paper. 
bioRxiv preprint doi: https://doi.org/10.1101/480798; this version posted November 28,2018 . The copyright holder for this preprint (which was not certified by peer review) is the author/funder, who has granted bioRxiv a license to display the preprint in perpetuity. It is made available under aCC-BY-ND 4.0 International license.

\section{Notch-Mediated Polarity Decisions in Mechanosensory Hair Cells}

\section{References}

1. S. Bray, Notch signalling: a simple pathway becomes complex. Nature reviews. Molecular cell biology. 7, 678-689 (2006).

2. S. J. Bray, Notch signalling in context. Nature reviews. Molecular cell biology. 9, 722-735 (2016).

3. M. Sjöqvist, E. R. Andersson, Do as I say, Not(ch) as I do: Lateral control of cell fate. Developmental Biology (2017), doi:10.1016/j.ydbio.2017.09.032.

4. A. J. Hudspeth, Integrating the active process of hair cells with cochlear function. Nature Reviews Neuroscience. 15, 600-614 (2014).

5. S. L. Shotwell, R. Jacobs, A. J. Hudspeth, Directional Sensitivity of Individual Vertebrate Hair Cells to Controlled Deflection of Their Hair Bundles*. Annals of the New York Academy of Sciences. 374, 1-10 (1981).

6. M. Montcouquiol et al., Identification of Vangl2 and Scrb1 as planar polarity genes in mammals. Nature. 423, 173-7 (2003).

7. H. López-Schier, A. J. Hudspeth, A two-step mechanism underlies the planar polarization of regenerating sensory hair cells. Proceedings of the National Academy of Sciences of the United States of America. 103, 18615-18620 (2006).

8. a Li, J. Xue, E. H. Peterson, Architecture of the mouse utricle: macular organization and hair bundle heights. Journal of neurophysiology. 99, 718-733 (2008).

9. A. Ghysen, C. Dambly-Chaudière, The lateral line microcosmos. Genes and Development. 21, 2118-2130 (2007).

10. T. Jiang, K. Kindt, D. K. Wu, Transcription factor $\mathrm{Em} \times 2$ controls stereociliary bundle orientation of sensory hair cells. eLife. 6, 1-26 (2017).

11. I. Mirkovic, S. Pylawka, A. J. Hudspeth, Rearrangements between differentiating hair cells coordinate planar polarity and the establishment of mirror symmetry in lateral-line neuromasts. Biology open. 1, 498-505 (2012).

12. E. Dow, A. Jacobo, S. Hossain, K. Siletti, A. J. Hudspeth, Connectomics of the zebrafish's lateral-line neuromast reveals wiring and miswiring in a simple microcircuit. eLife Sciences. 7, e33988 (2018).
13. M. Boareto et al., Jagged-Delta asymmetry in Notch signaling can give rise to a Sender/Receiver hybrid phenotype. Proceedings of the National Academy of Sciences of the United States of America. 112, E402-9 (2015).

14. D. Sprinzak et al., Cis-interactions between Notch and Delta generate mutually exclusive signalling states. Nature. 465, 86-90 (2010).

15. M. Gray, C. B. Moens, S. L. Amacher, J. S. Eisen, C. E. Beattie, Zebrafish deadly seven Functions in Neurogenesis. Developmental Biology. 237, 306-323 (2001).

16. A. D. Kim et al., Discrete Notch signaling requirements in the specification of hematopoietic stem cells. The EMBO Journal. 33, 2363-2373 (2014).

17. A. Amsterdam et al., Identification of 315 genes essential for early zebrafish development. PNAS. 101, 12792-12797 (2004).

18. D. Jülich et al., beamter/deltaC and the role of Notch ligands in the zebrafish somite segmentation, hindbrain neurogenesis and hypochord differentiation. Developmental Biology. 286, 391-404 (2005).

19. J. Ezan et al., Primary cilium migration depends on G-protein signalling control of subapical cytoskeleton. Nature cell biology. 15, 1107-15 (2013).

20. B. Tarchini, C. Jolicoeur, M. Cayouette, A molecular blueprint at the apical surface establishes planar asymmetry in cochlear hair cells. Developmental cell. 27, 88-102 (2013).

21. L. Jiang, A. Romero-Carvajal, J. S. Haug, C. W. Seidel, T. Piotrowski, Gene-expression analysis of hair cell regeneration in the zebrafish lateral line. PNAS. 111, E1383-E1392 (2014).

22. I. Wibowo, F. Pinto-Teixeira, C. Satou, S. Higashijima, H. López-Schier, Compartmentalized Notch signaling sustains epithelial mirror symmetry. Development (Cambridge, England). 138, 1143-52 (2011). 